\title{
ANDROID OS BASED WIRELESS DATA ACQUISITION SYSTEM VIA BLUETOOTH
}

\author{
Gurpal Singh ${ }^{1}$, Inderpal Singh ${ }^{2}$ \\ ${ }^{1}$ IC Design Engineer, Broadcom India Private Ltd, Karnataka, India \\ ${ }^{2}$ Student, Department of Electronics and Comm. Engg., GNDEC Ludhiana, Punjab, India
}

\begin{abstract}
Data acquisition is an important part of any type of instrumentation. New technology can be used make data acquisition system more portable and powerful. Android mobile operating system which is open source and widely acceptable, can be used make a Data Acquisition System, which is not only portable but also powerful and open source. This will also make the study of electronics more advanced and interesting in technical institutes.
\end{abstract}

Keywords: Android, Bluetooth, Data Acquisition, Engineering Education and Microcontroller

\section{INTRODUCTION}

This document describes the designing of prototype model of wireless data acquisition system using Android Operating system. Some years ago, when a device with $1-2 \mathrm{GHz}$ processor, 1-2GB RAM was discussed, the device name was "Personal Computer". But in present, when someone talks about same specifications, the device name is "Samrtphone". As technology has evolved, mobile phones have become more users friendly and can do much more tasks than simply making calls. Smartphones today not only give high processing capabilities, in addition to this they offer features like Accelerometer, Gyro Sensor, RGB Sensor, Proximity Sensor, Barometer, Compass and connectivity options like Bluetooth, DLNA, USB, Wifi etc., depending upon device to device and manufacturing companies.

The smartphone users are increasing rapidly. These smartphones can be used in many innovative ways. Using smartphone for data acquisition is one of the innovative ways to make it portable and powerful. Data acquisition is the process in which real worlds physical conditions, which are measured by signals, are sampled and are then converted to digital one so that they can be processed by digital system like computer and in our case smartphone. Practical data acquisition system consists of many parts like transducer, amplifiers, filters, A-to-D convertor, software etc [1]. But, as this work is for designing a prototype system, it deals only with the A-to-D convertor and software part. Testing signals are taken by using signal generator.

A data acquisition system can be divided into two parts: Hardware and Software. Hardware section consists of the microcontroller, which converts the analog signal to digital one and sends it to Android phone via Bluetooth. Software section includes developing Android application which receives the data via Bluetooth and saves the digital values. As the system is wireless and use Bluetooth mode of communication, discussion about Bluetooth will also be given in this paper

\section{DETERMINATION OF TESTING PLATFORM}

The main goals of this project are:

- Designing smartphone application

- Designing a hardware module, that is capable of analog to digital conversion.

- Both hardware and smartphone can communicate with each other using Bluetooth channel.

For designing smartphone application, Android Mobile OS is chosen and for designing hardware module ATmega8 microcontroller. The reasons for these selections are explained below:

\subsection{Selection of Android Mobile OS}

A mobile operating system, also referred to as mobile OS, is the operating system that operates a smartphone, tablet, PDA or other digital mobile devices. The main requirements of the mobile OS for this project are:

- $\quad$ Bluetooth Support

- Openness

- Accessibility

- Low complexity

Bluetooth support is one of the main requirements of this project. Mobile OS should allow the third party applications to install and access the mobile resources. For the research purpose, it should be low cost and well documented.

Android has powerful APIs, excellent documentation, a thriving developer community, and no development or distribution costs. In addition to this, it has following advantages:

- Open source and free

- There is no approval process for application distribution. 
- Wide selection of hardware,

- $\quad$ Easily available Tutorials and Developing tools

There is one more reason for choosing Android i.e. government policy to distribute "Aakash" tablets at low price to students. This will further decrease the cost of virtual instrumentation for students and creates good learning environment.

\subsection{Selection of ATmega8}

Microcontroller is a self contained small computer on single integrated chip in which a processor, support, memory, and input/output are all contained in a single package. In the market there are number of manufactures with different type of microcontroller, with different features and architecture are available. The main requirements of microcontroller in this project are:

- UART Support

- ADC Support

- Accessibility

- Low Complexity

The type of communication over the Bluetooth, which is going to be used in this project, is serial communication, so microcontroller should support UART. In addition to this, the basic need of this hardware part of this project is to convert analog signal into digital. To full fill this requirement, $\mathrm{ADC}$ is compulsory.

ATmega8 has inbuilt oscillator of $1 \mathrm{MHz}$, USART support and ADC convertor. This decreases the external component count and hence overall cost and power consumption. In addition to this, ATmega8 has feature of ISP programming, which decreases the developing and testing time of prototype project. It is a high-performance, low-power AVR 8-bit microcontroller with advanced RISC architecture. It has $8 \mathrm{~K}$ Bytes of In-System Self-programmable Flash program memory, 512 Bytes EEPROM and $1 \mathrm{~K}$ Byte Internal SRAM.

\subsection{Selection of Bluetooth Module}

Smartphones powered by Android mobile OS supports Bluetooth communication. The most common type of Bluetooth socket is RFCOMM, which is the type supported by the Android APIs. RFCOMM is a connection-oriented, streaming transport over Bluetooth. It is also known as the Serial Port Profile (SPP) [2]. On the other hand ATmega8 does not support Bluetooth communication. To transfer data between Android smartphone and ATmega8 microcontroller, an external module is needed, which has feature of transferring data using Bluetooth. In addition to this, it should also support SPP. For this purpose, module used in this project is HC-05. HC-05 series modules can be used in either mode i.e. Master and Slave mode [3]. Bluetooth module has a feature of serial communication with $9600-115200 \mathrm{bps}$ [4]. It operates in the frequency range 2.4-2.524 GHz. It also has built-in antenna. Although HC-05 has total 32-pins, only 7 pins are used for interfacing in this project. The interfacing circuit is shown in Fig. 1

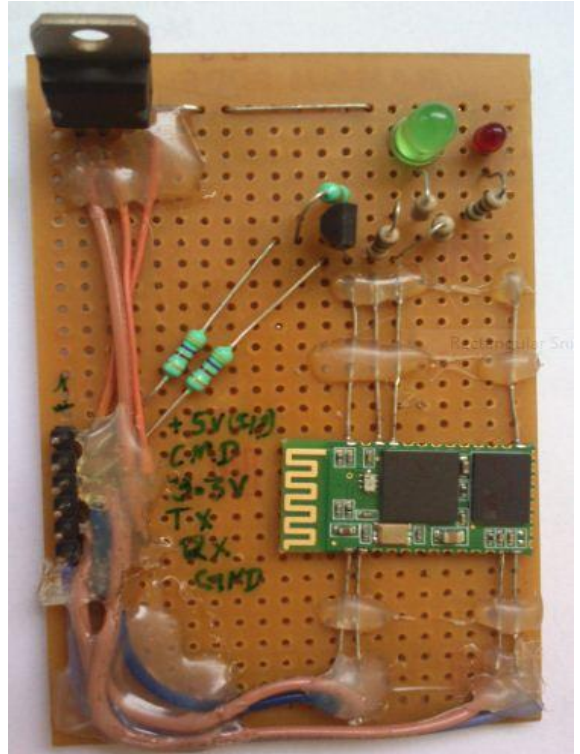

Fig -1: HC-05 interfacing Circuit

As shown in figure, the interfacing circuit has six pins for interfacing. As the circuit is not a standard circuit and specifically designed for this project, the function of each pin is given below:

- $\quad$ Pin 1: This pin is like the VCC of this circuit. It is connected to input pin of AZ1117-3.3 voltage regulator. The maximum optimal input voltage can be $15 \mathrm{~V}$ [5]

- $\quad$ Pin 2: This pin is command pin (CMD). This pin controls the voltage level at Pin 34 of HC-05. Logic value on this pin

$$
\begin{aligned}
& \text { ' } 0 \text { ': AT Mode } \\
& \text { ' } 1 \text { ': Communication mode }
\end{aligned}
$$

- $\quad$ Pin 3: This pin gives 3.3V output. This output can be use to power any external circuit, which can work at $3.3 \mathrm{~V}$

- $\quad$ Pin 4: This pin is directly connected to TX Pin of HC-05

- Pin 5: This pin is directly connected to RX of HC05

- Pin 6: This is the Ground pin of the application circuit.

The default password of HC-05 is " 1234 ", which should be entered during pairing with Android mobile phone.

\section{ARCHITECTURE AND IMPLEMENTATION DETAILS}

This section can be divided into two parts; one is Hardware and second is Software. The requirements, architecture and implementation details of each module are given below:

\subsection{Hardware Part}

The main requirement of data acquisition hardware module in this project is to convert the analog signal to digital signal and then send it to Android Smartphone via Bluetooth 
connection. The architecture used in this project is given in Fig. 2

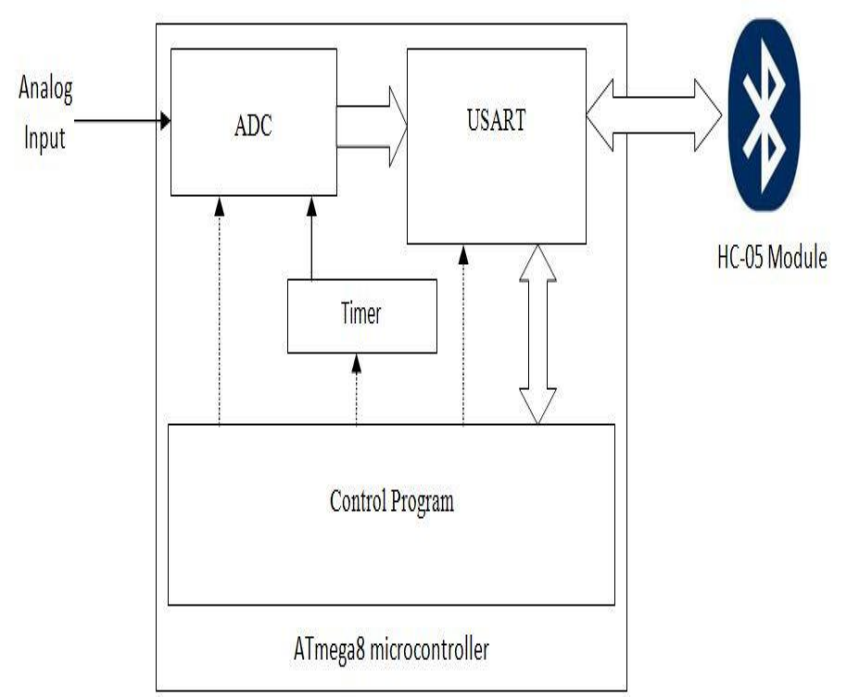

Fig -2: Architecture of Hardware Module

The function of control program is to control the functioning and initialization of USART, ADC and Timer of microcontroller. Timerl is set to work in CTC mode i.e. Clear Timer on Compare mode to generate interrupt after every $2 \mathrm{~ms}$. In the interrupt service routine i.e. ISR of Timer1, analog to digital conversion is done by ADC which is set to 8-bit resolution and then converted digital values are send to HC-05 via USART. Thus, this prototype data acquisition system has sampling rate of $500 \mathrm{~Hz}$. USART is set to work at 9600 baud rate with 1 stop bit and 8 data bits setting.

Control program also controls the communication between ATmega8 and Android smartphone. Before starting conversion and sending digital values, it will continuously send connection request to Bluetooth device after every $100 \mathrm{~ms}$. After acceptance of the request, it will start ADC conversion and sends data after every $2 \mathrm{~ms}$. The complete setup of Hardware module is shown in Fig. 3

As shown in fig. 3, dual supplies are used; one is $5 \mathrm{~V}$ and second is $3.3 \mathrm{~V}$. As said earlier, this is a prototype project and it needs programming the ISP again and again. Programming in our case is done by using Lpt 1 port of PC and it needs microcontroller to be powered with $5 \mathrm{~V}$ power supply. But our actual project operates on $3.3 \mathrm{~V}$, to save the power. Hence, dual power supplies are used. Switch button is used to toggle between two supplies, white led indicate $5 \mathrm{~V}$ and green led indicates $3.3 \mathrm{~V}$ Two leds, which are adjacent to microcontroller, green and red, are used to indicate the operating state of microcontroller.

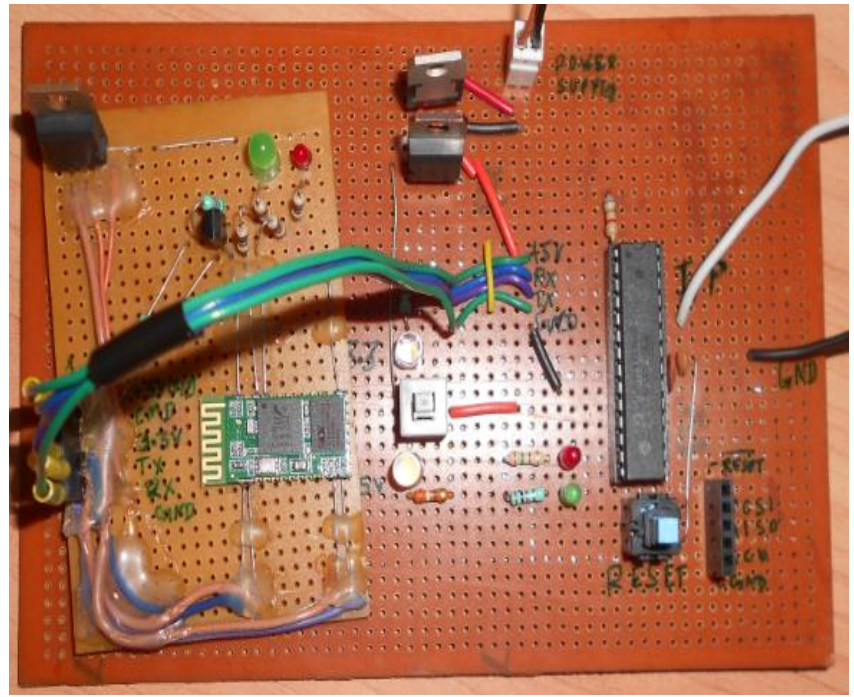

Fig -3: Hardware Module Circuit

\subsection{Software Part}

Use cases have been specified for the mobile application, to get a sense of the behavior of the application. Fig. 4 gives an overview of the use cases defined.

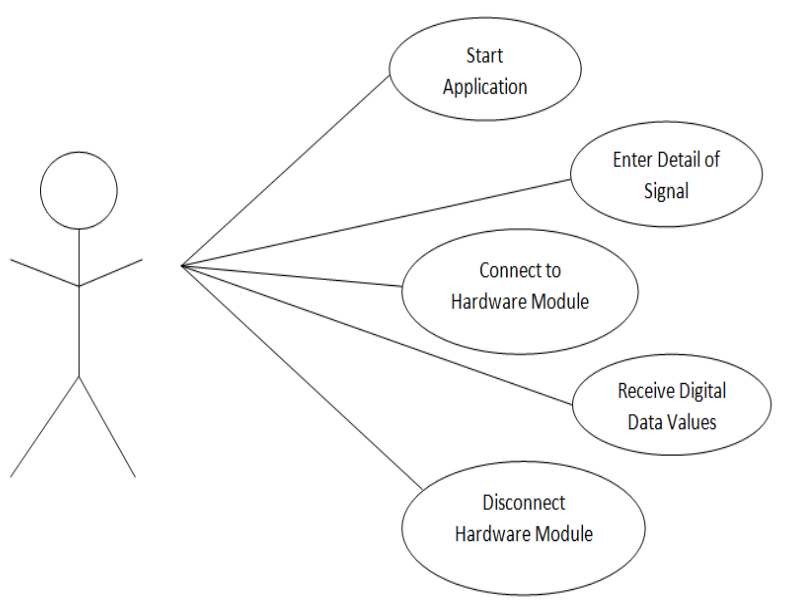

Fig -4: Use Case Diagram of Application

The user starts mobile application by clicking on the application icon. After starting the application, user can enter information of signal, date and time of taking signal etc. using the SQLite Database Adatper. Furthermore user can connect to hardware module via Bluetooth connection. After connecting to the hardware module, mobile application starts receiving digital values of analog signal. The received data will then be saved in a text file. The application can be disconnected from hardware module if there not any data to receive. If there is need to plot the graph of received data for better visualization, it can be done using free and open source library "achartengine-1.0.jar" [6]. The architecture on which this application is designed is given in Fig. 5. 


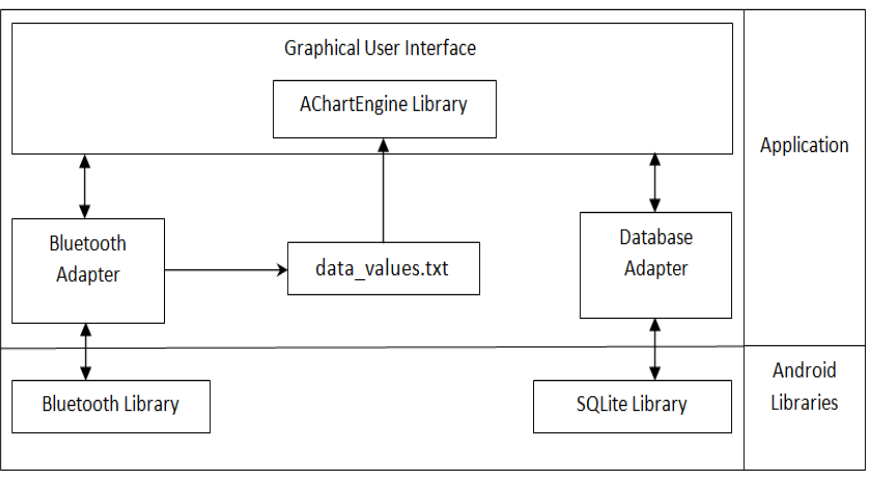

Fig -5: Architecture of Android Application

The main android libraries which are used in this project are Bluetooth Library and SQLite Library. To interact with these libraries, two adapters are used; one is Bluetooth Adapter and second is Database Adapter. Bluetooth Adapter takes care of making connection with hardware module, receiving data and saving it to "data_values.txt" file. The text file is saved in the external memory of android system. The file can be accessed by another application and can be transferred to PC easily, due to its location i.e. external memory [2]. Database Adapter takes the input from the user, about the signal and save it in the Android System. This data is not accessible by another application. AChartEngine library helps for plotting data values. Data values are now stored in text file, and can be used for further calculations.

\subsection{Programming Details}

The programming of hardware module that is Atmega8 microcontroller is done using the embedded $\mathrm{C}$. The data_acq.apk is written using JAVA and XML codes. XML code is use to design the layout of the activity and JAVA code is use to design the functionality of the system i.e. to save signal information, making Bluetooth connection and saving received values.

\section{TESTING}

In this Project, the black box testing is used to carry out the mobile application testing for the development work. Black Box testing is described as a method of software testing that is focused on functionalities of an application. It is defined as a software testing technique whereby the internal structures (workings) of the item under test are not known to the tester. [7]

The minimum requirement for the smartphone is, it should be loaded with Android 2.3.3-2.3.7 Gingerbread (API Level 10). The test carried out extends from installation on Android Phone, and Bluetooth functionalities like connectivity to reading ECG.

Data Acquisition application is tested on three devices: Sony Xperia Go, SAMSUNG Galaxy Lite Duos and HTC Explorer, all with different price range and manufacturing company. One thing which was common in all was that all run on Android OS. All the testing applications run on these devices very well, without any manufactures or brand issue. Testing signals are taken from signal generator.

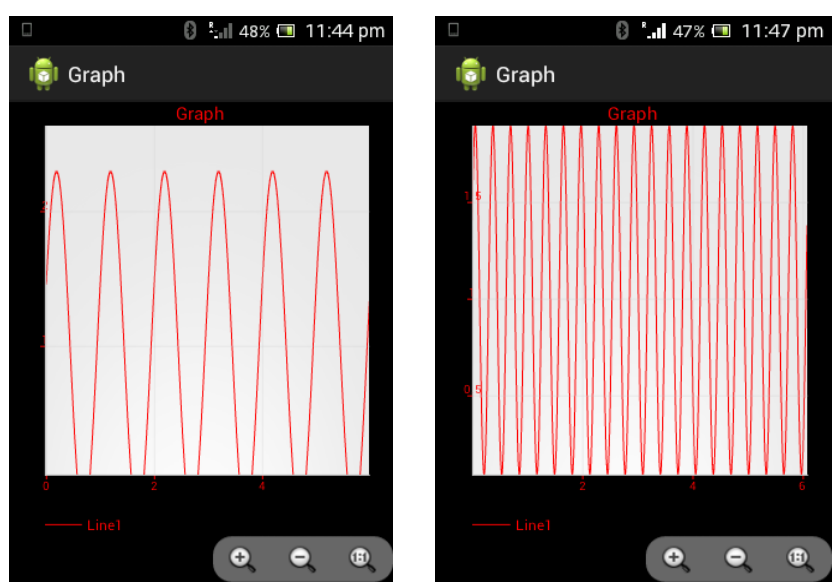

Fig -6: Testing Signals

Fig. 6(a) shows the graph plotted on smartphone for sine wave with amplitude $1.3 \mathrm{~V}$, offset $1 \mathrm{~V}$ and frequency $1 \mathrm{~Hz}$ and Fig. 6(b) shows output for amplitude $0.9 \mathrm{~V}, 1 \mathrm{~V}$ offset and $3 \mathrm{~Hz}$ frequency. $1 \mathrm{~V}$ offset is used because, ADC cannot convert negative voltages. The negative part of the signal in Fig 1(a), is clipped off.

\section{EXPERIENCE AND LESSON LEARNED}

In this paper, we presented a prototype project to show how android OS and device can be used for data acquisition system. This project is only the prototype and lots more options can be add like doing calculations, sending data to $\mathrm{PC}$ for doing calculations, which required more processing power.

Before this project, we have developed only microcontroller based project. We had no experience in mobile application development and at Java programming. But when we start reading tutorial and android documentation provided by Google [2] itself, we find it very easy to develop for android. Also some books on android [8, 9] and java programming were very helpful.

There were lots of new things to learn while developing. As the development is open source, we found solutions of problems very easily, which we get during developing. Also tutorials on achartengine-1.0.0.jar library were very helpful.Paragraph comes content here. Paragraph comes content here.

\section{CONCLUSIONS}

The main goal of this paper is to implement data acquisition system using an Android device which today has high processing capability and lots more inbuilt features. Android OS has many advantages like free of cost, wide range of devices available, easily available tutorials, which make this ideal for a student for developing projects. All the tools used are open source and free of charge. 
The main advantages of designing data acquisition system using Android OS are the user defined graphical interface and versatility, which make it easy to use and learn. VI approach along with android device shortens the developing time for students and overall system cost. .

\section{REFERENCES}

[1]. Data Acquisition: An Introduction (Last accessed Oct 2013) [Online], http://www.alldatasheet.com/datasheetpdf/pdf/137196/BCDSEMI/AZ1117.html

[2]. Android developers (Last accessed Sept 2013) [Online], http://developer.android.com

[3]. HC Serial Bluetooth (Last accessed Oct 2013) [Online], Available: http://www.mcu-turkey.com/wpcontent/uploads/2013/01/HC-Serial-Bluetooth-Products201104.pdf

[4]. Jianping CAI et al., -A Bluetooth Toy Car Control Realization by Android Equipmentll, 2011 International Conference on Transportation, Mechanical, and Electrical Engineering, Changchun, 16-18 Dec. 2011, pp. 2429-2432

[5]. AZ1117 Datasheet (Last Accessed Oct 2013) [Online], Available: http://www.alldatasheet.com/datasheetpdf/pdf/137196/BCDSEMI/AZ1117.html

[6]. Achartengine (Last Accessed Nov 2013) [Online], Available: http://www.achartengine.org

[7]. Mumtaz Ahmad Khan, Mohd. Sadiq -Analysis of Black Box Software Testing Techniques: A Case Studyll, 2011 International Conference and Workshop on Current Trends in Information Technology (CTIT), IEEE, Dubai, October 26-27, 2011, pp. 1-5

[8]. Reto Meier, -Professional Android ${ }^{\mathrm{TM}} 4$ Application Developmentll, 4th ed., John Wiley \& Sons, Inc., 2012

[9]. Sayed Y.hashimi, Satya Komatineni, Pro Android [M], 1st ed., Apress, 2009

\section{BIOGRAPHIES}

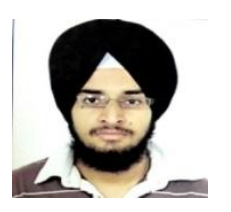

Gurpal Singh has received his Bachelor degree from GNDU, Amritsar in 2011 and Master degree from IIT (BHU) in 2013. Currently he is working as IC Design Engineer in Broadcom India Private Ltd.

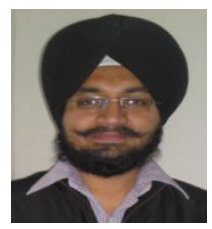

Inderpal Singh has received his Bachelor degree from SBSCET, Ferozpur (now State Technical Campus) in 2008. Currently pursuing M.Tech from GNDEC, Ludhiana 